${ }^{1}$ Supplementary Information for

\title{
Tweaking Palladium Electronic Structure to Attain Oxygen Reduction Activity Superior to Platinum/C
}

Saurav Ch. Sarma, ${ }^{[a, b]}$ Sai Manoj Kaja, ${ }^{[a, b]}$ Vidyanshu Mishra, ${ }^{[a, b]}$ Chathakudath

Prabhakaran Vinod ${ }^{[c]}$ and Sebastian C. Peter *[a,b]

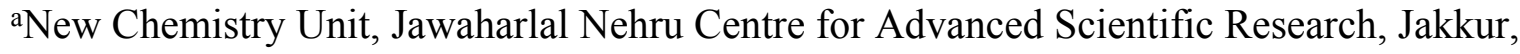
Bangalore-560064, India.

bSchool of Advanced Materials, Jawaharlal Nehru Centre for Advanced Scientific Research, Jakkur, Bangalore-560064, India.

${ }^{\mathrm{c}}$ Catalysis Division, CSIR-National Chemical Laboratory,Dr. Homi Bhabha Road, Pune 411 008, India

*Corresponding author: sebastiancp@jncasr.ac.in. Phone: 080-22082998, Fax: 08022082627 


\section{Synthesis of catalysts:}

\section{Chemicals used:}

Following chemicals are used in the synthesis protocol. Palladium acetylacetonate $\left(\operatorname{Pd}(\mathrm{acac})_{2}\right.$, Sigma-Aldrich 99.99\%), Europium (III) chloride $\left(\mathrm{EuCl}_{3}\right.$, Sigma-Aldrich 99.99\%), Ytterbium (III) chloride $\left(\mathrm{YbCl}_{3}\right.$, Sigma-Aldrich 99.99\%), Ethylene Glycol (EG, 99\%), Sodium Borohydride $\left(\mathrm{NaBH}_{4}\right.$, Sigma-Aldrich 98\%), Ethanol (99.9\%). All the air-sensitive compounds are handled in glove box.

\section{Synthesis procedure:}

In a typical procedure, $\mathrm{YbCl}_{3}(0.1 \mathrm{mmol}, 27.9 \mathrm{mg}), \mathrm{EuCl}_{3}(0.1 \mathrm{mmol}, 25.8 \mathrm{mg}) . \mathrm{Pd}(\mathrm{acac})_{2}(0.3$ mmol, $97.92 \mathrm{mg}$ ) was weighed in stoichiometric quantities in order to prepare the following alloys: $\mathrm{RE}_{0.1} \mathrm{Pd}_{0.9}(\mathrm{RE}=\mathrm{Yb} \& \mathrm{Eu}, \mathrm{EuPd}-\mathrm{A} \& \mathrm{YbPd}-\mathrm{A})$. A small amount $(\sim 1$ to $2 \mathrm{~mL})$ of EG was added to prevent hydrolysis of the added salts. $\mathrm{NaBH}_{4}$ was added to reduce the Pd salts to their corresponding elemental zero oxidation states. 15 to $16 \mathrm{~mL}$ of EG was added to form the reaction solution. The solution turned black due to the reduction of the corresponding $\mathrm{Pd}^{2+}$ to its elemental zero oxidation state. The contents were homogeneously mixed using an orbital shaker and heated to $220^{\circ} \mathrm{C}$ for $24 \mathrm{~h}$. The precipitate was centrifuged and washed repeatedly with an ethanol. The obtained products were then vacuum dried at $60{ }^{\circ} \mathrm{C}$ and used for further characterizations. Quantities were doubled by keeping the solvent volume same for preparing bimetallic REPd compounds (EuPd-B \& YbPd-B).

\section{Characterization:}

The PXRD measurements at room temperature were carried out on a Rigaku miniflex X-ray diffractometer with $\mathrm{Cu}-K \alpha$ as the $\mathrm{X}$-ray source $(\lambda=1.5406 \AA)$. The instrument is equipped with a position sensitive detector in the angular range $20^{\circ} \leq 2 \theta \leq 90^{\circ}$ 
with the step size $0.02^{\circ}$ and scan rate of $1 \mathrm{sec} / \mathrm{step}$ calibrated against corundum standard. The experimental patterns were compared to the pattern simulated from the database.

Quantitative microanalyses on all the samples were performed with an Gemini-SEM 500 instrument equipped with an AztecOne (Oxford instruments) instrument. Data were acquired with an accelerating voltage of $5 \mathrm{kV}$ (image acquisition), $20 \mathrm{kV}$ (EDS analysis) and a $100 \mathrm{~s}$ accumulation time. The EDS analysis was performed using P/B-ZAF standardless method (where, $\mathrm{Z}=$ atomic no. correction factor, $\mathrm{A}=$ absorption correction factor, $\mathrm{F}=$ fluorescence factor, $\mathrm{P} / \mathrm{B}=$ peak to background model) on selected spots and points.

TEM images and elemental mapping were collected using a TECHNAI TEM instrument equipped with $\mathrm{EDAX}{ }^{\circledR}$. The samples for these measurements were prepared by sonicating the nanocrystalline powders in ethanol and drop-casting a small volume onto a carbon-coated copper grid.

XPS measurements were carried out using Thermo K-alpha ${ }^{+}$spectrometer using micro focused and monochromated $\mathrm{Al} \mathrm{K} \alpha$ radiation with energy $1486.6 \mathrm{eV}$. The pass energy for spectral acquisition was kept at $50 \mathrm{eV}$ for individual core-levels. The electron flood gun was utilized for providing charge compensation during data acquisition. Further, the individual core-level spectra were checked for charging using $\mathrm{C} 1 \mathrm{~s}$ at $284.6 \mathrm{eV}$ as standard and corrected if needed. The peak fitting of the individual core-levels were done using Fityk software with a Shirley type background.

\section{Electrochemical measurements:}

All the electrochemical measurements were carried out using a CHI760E electrochemical workstation with three electrode channels at room temperature. A conventional RDE and RRDE electrodes are used as a working electrode, graphite rod as a counter electrode and $\mathrm{Hg} / \mathrm{HgO}(\mathrm{MMO})$ as a reference electrode were used. 
Before all the measurements, the electrolyte was de-aerated with continuous purging of nitrogen gas for $30 \mathrm{~min}$. The catalyst ink was prepared by dispersing $2 \mathrm{mg}$ of catalyst in $200 \mu \mathrm{L}$ of mixed solvent solution (IPA: $\mathrm{H}_{2} \mathrm{O}=1: 3 \mathrm{v} / \mathrm{v}$ ). Nafion solution (5 $w t \%$, Sigma Aldrich) is diluted with isopropyl alcohol (IPA) to $0.1 \mathrm{wt} \%$. From the prepared catalyst ink, a $10 \mu \mathrm{L}$ of the slurry was dropcasted on GC electrode. Upon drying, $2 \mu \mathrm{L}$ of $0.1 \mathrm{wt} \%$ of Nafion solution have been added to the dropcasted electrode and dried overnight in air. The GC electrode was polished with $0.05 \mu \mathrm{m}$ alumina slurry and washed several times with distilled water prior to the deposition of catalyst slurry. As-synthesized Pd/C and Pt/C (20 wt\%, Sigma Aldrich) (with same $\mathrm{Pd} / \mathrm{Pt}$ loading on electrode) was used for comparison of activity with same loading as the synthesized catalysts. Linear sweep voltammetry (LSV) was recorded with a sweep rate of $5 \mathrm{mV} / \mathrm{s}$ in $0.1 \mathrm{M} \mathrm{KOH}$ electrolyte solution under steady state conditions. Tafel plots (TP) were derived from LSV measurement at different rpms. Accelerated degradation test (ADT) was performed with graphite rod (purity $>99.9$ $\%$, sigma aldrich) to avoid $\mathrm{Pt}$ dissolution. Stability tests of were carried out at a scan rate of $100 \mathrm{mVsec}^{-1}$ and within a potential window of $0.6-0.9 \mathrm{~V}$ vs. RHE. Potential window for the stability test was selected based on the previous literatures. Stability of the catalysts were checked using RDE measurements in a $3 \mathrm{~mm}$ diameter electrode. LSV graphs were recorded before the accelerated degradation test (ADT), after 1000 cycles and after 5000 electrochemical cycles to check the stability of the catalyst. Assessment of the selectivity towards $\mathrm{H}_{2} \mathrm{O}$, before and after the ADT process, was checked using RRDE electrode with $4 \mathrm{~mm}$ diameter and keeping all 
other experimental conditions similar. All the electrochemical data, presented in this work, are referenced to RHE scale. All the ORR electrochemical evaluations were performed at $1600 \mathrm{rpm}$.

\section{Calibration of $\mathrm{Hg} / \mathrm{HgO}$ w.r.t RHE in $0.1 \mathrm{M} \mathrm{KOH}$ and at a scan rate of $5 \mathrm{mV} / \mathrm{sec}:$}

The calibration of $\mathrm{Hg} / \mathrm{HgO}$ electrode was performed in a standard three-electrode system with polished Pt wires as the working and counter electrodes, and the $\mathrm{Hg} / \mathrm{HgO}$ as the reference electrode. Electrolytes are continuously purged and saturated with high purity $\mathrm{H}_{2}$. Cyclic voltammetry $(\mathrm{CV})$ is then run at a scan rate of $5 \mathrm{mVsec}^{-1}$, and the average of the potential, from both the sweeps, at which the current crossed zero is taken to be the thermodynamic potential for the hydrogen electrode reactions. For example, in $0.1 \mathrm{M} \mathrm{KOH}$, the zero current point is at $-0.936 \mathrm{~V}$, so $\mathrm{E}_{(\mathrm{RHE})}=\mathrm{E}_{(\mathrm{Hg} / \mathrm{HgO})}+0.936 \mathrm{~V}$.

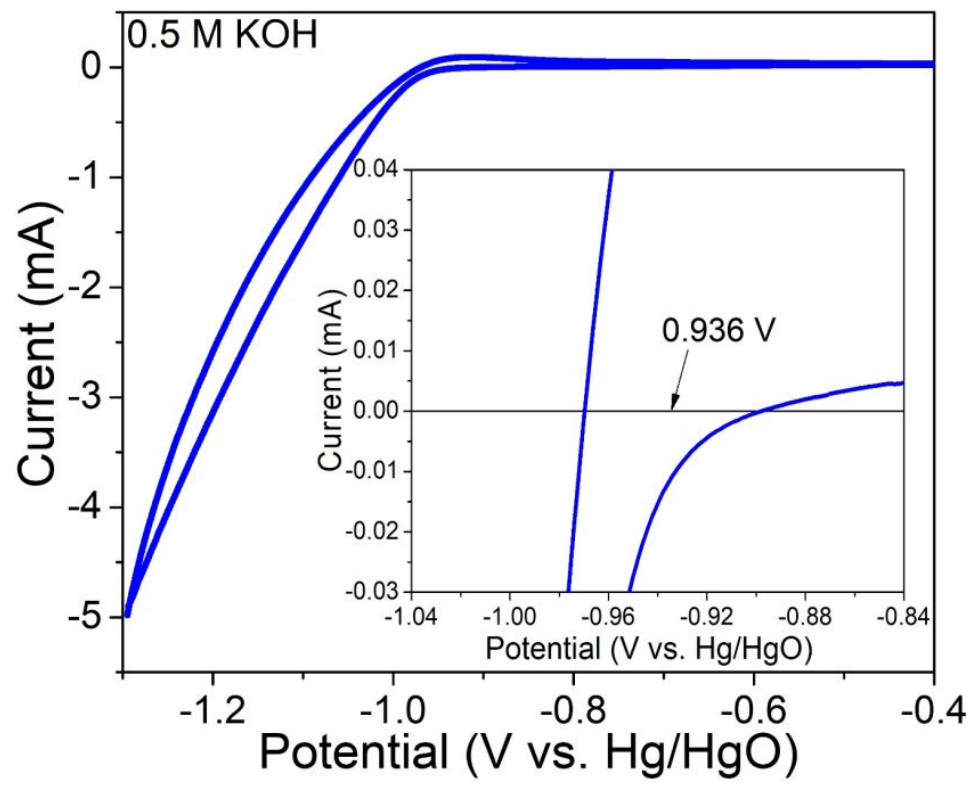

$\underline{\text { Derivation of } \mathrm{H}_{2}} \underline{\mathrm{O}}_{2} \underline{\text { and } \mathrm{H}_{2}} \underline{\underline{O} \text { percentage: }}$ 
$\mathrm{H}_{2} \mathrm{O}_{2}$ percentage were derived from the RRDE calculation. The amount of $\mathrm{H}_{2} \mathrm{O}_{2}$ was then calculated from the ring and disk current using the following formula:

$$
\chi_{H 202}=\frac{2 \frac{I_{R}}{N}}{I_{D}+\frac{I_{R}}{N}}
$$

where $\chi_{\mathrm{H} 2 \mathrm{O} 2}$ is the number of moles of $\mathrm{H}_{2} \mathrm{O}_{2}$ formed per mole of $\mathrm{O}_{2}, \mathrm{I}_{\mathrm{R}}$ is the ring (collection electrode) current and $\mathrm{I}_{\mathrm{D}}$ is the disc (working electrode) current.

\section{Tables}

Table S1. The table shown lists the particle size of the catalysts determined from Scherrer's equation.

\begin{tabular}{|c|c|c|c|c|}
\hline Catalyst & FWHM & Theta (in angle) & Crystallite Size (nm) & $\begin{array}{c}\text { Lattice } \\
\text { Strain }\end{array}$ \\
\hline YbPd-A & 0.3473046 & 20.0674 & 24.3 & 0.009 \\
\hline EuPd-A & 0.2892461 & 20.086685 & 29.2 & 0.007 \\
\hline YbPd-B & 0.4178431 & 19.9085 & 20.2 & 0.0104 \\
\hline & 0.3253861 & 20.1231 & 25.9 & 0.008 \\
\hline EuPd-B & 0.2899682 & 19.835645 & 29.1 & 0.007 \\
\hline & 0.199047 & 20.17805 & 42.4 & 0.005 \\
\hline
\end{tabular}

Table S2. Relative composition of $\mathrm{Pd} / \mathrm{PdO}$ as determined from XPS.

\begin{tabular}{|c|c|}
\hline Catalysts & Pd/PdO \\
\hline YbPd-A & 1.30 \\
\hline YbPd-B & 1.27 \\
\hline EuPd-A & 1.51 \\
\hline EuPd-B & 1.04 \\
\hline
\end{tabular}

Table S3. The table shown lists the average atomic percentage and average weight percentage of the catalyst obtained from the EDAX measurements. 


\begin{tabular}{|c|c|c|}
\hline Catalyst & Average At \% & Average Wt\% \\
\hline YbPd-A & $\mathrm{Yb}=7.4 \%, \quad \mathrm{Pd}=92.6 \%$ & $\mathrm{Yb}=11.5 \%, \mathrm{Pd}=88.5 \%$ \\
\hline EuPd-A & $\mathrm{Eu}=12.6 \%, \mathrm{Pd}=87.4 \%$ & $\mathrm{Eu}=16.1 \%, \mathrm{Pd}=83.9 \%$ \\
\hline
\end{tabular}


Table S4. Hirshfeld charge analysis of REPd, $\mathrm{Pd}$ and Pt before and after $\mathrm{O}_{2}$ adsorption. Orange colored column indicates the change in charge before and after the adsorption process.

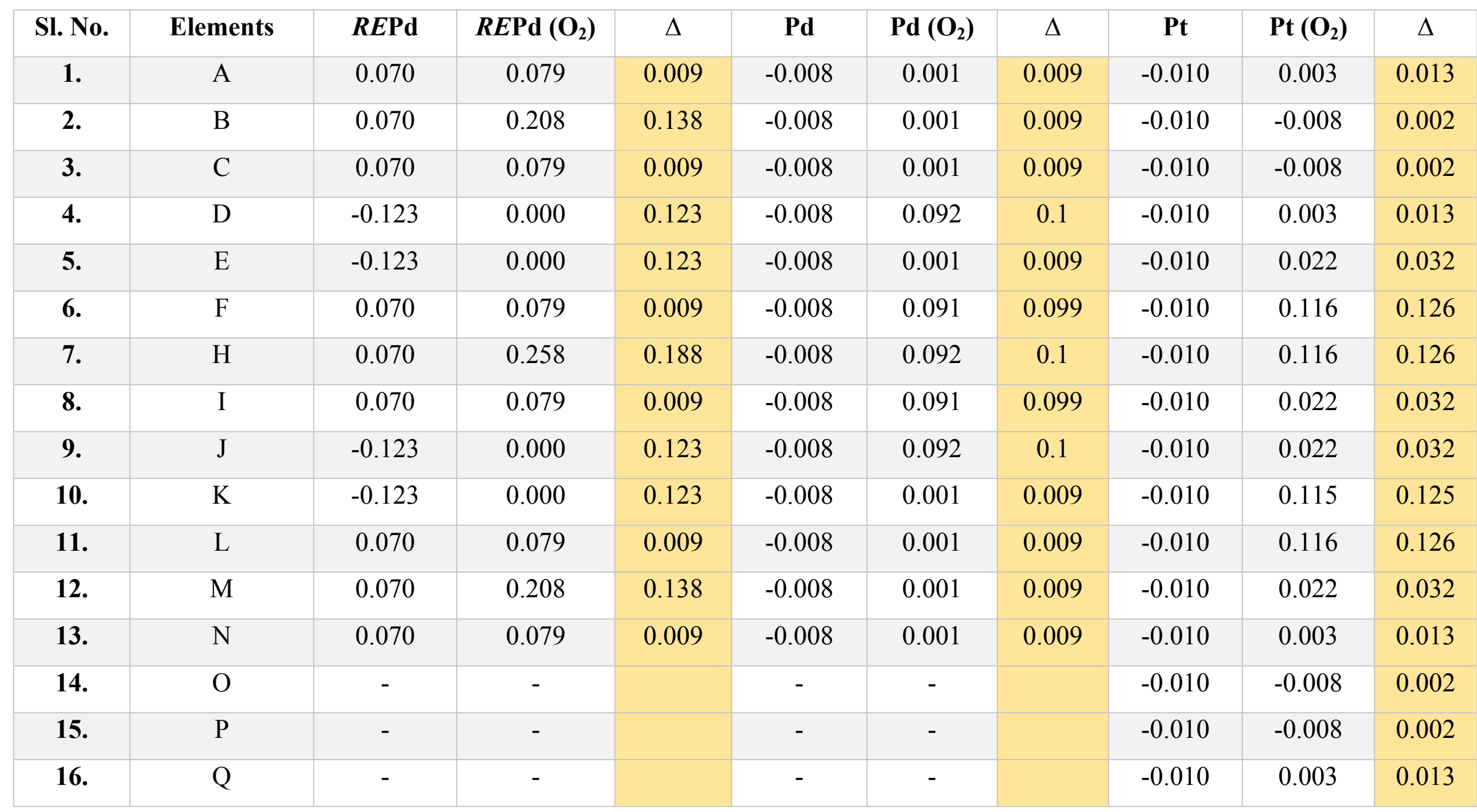




\section{Figures:}

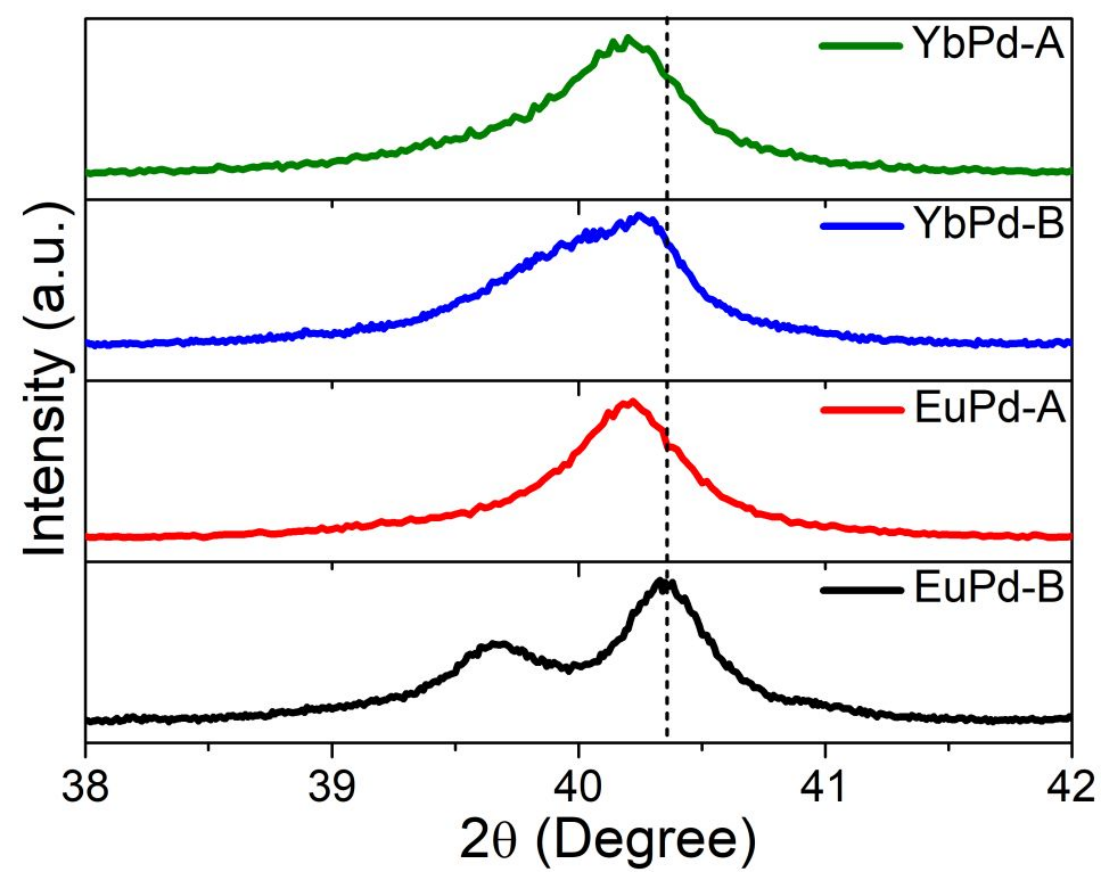

Figure S1. Comparison of zoomed experimental PXRD pattern of EuPd-A, YbPd-A, EuPd-B and $\mathrm{YbPd}-\mathrm{B}$.

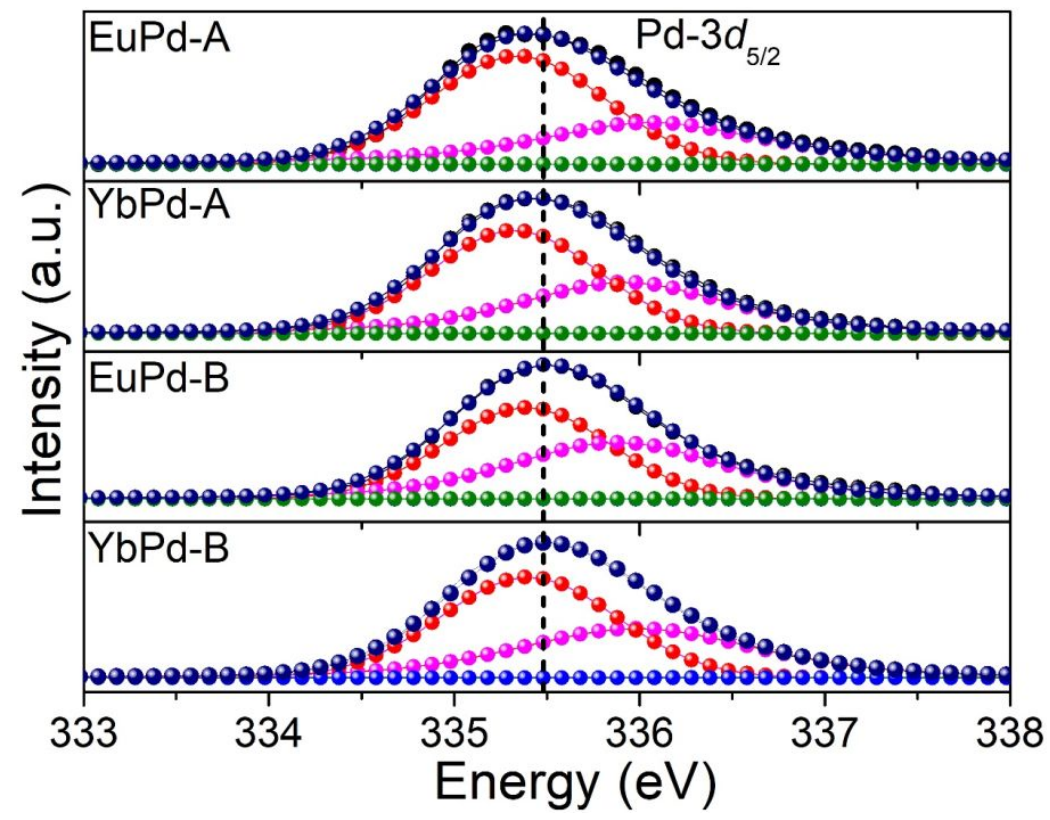

Figure S2. Comparison of high resolution Pd- $K$ edge XPS spectra of $R E P d$ catalysts showing the presence of PdO at $336.2 \mathrm{eV}$. Blue represents overall fit of the XPS spectra, red and pink color represents the deconvoluted peaks and green represents the residual spectra. 


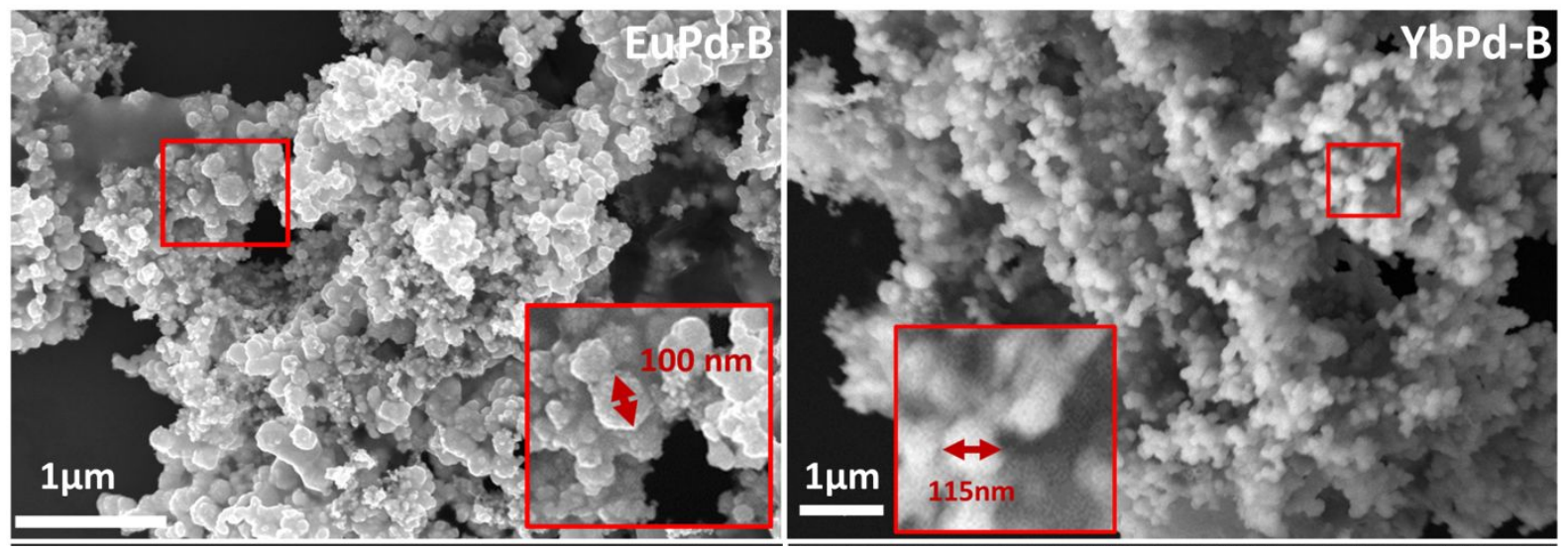

Figure S3. FE-SEM images of EuPd-B (left) and YbPd-B (right) showing hierarchical nature of the nanoparticles.

(a)

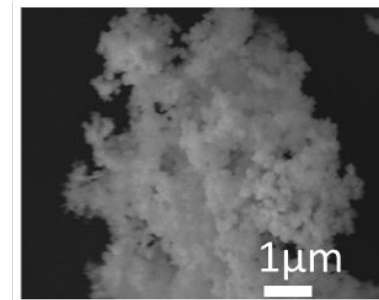

(2)

(3)
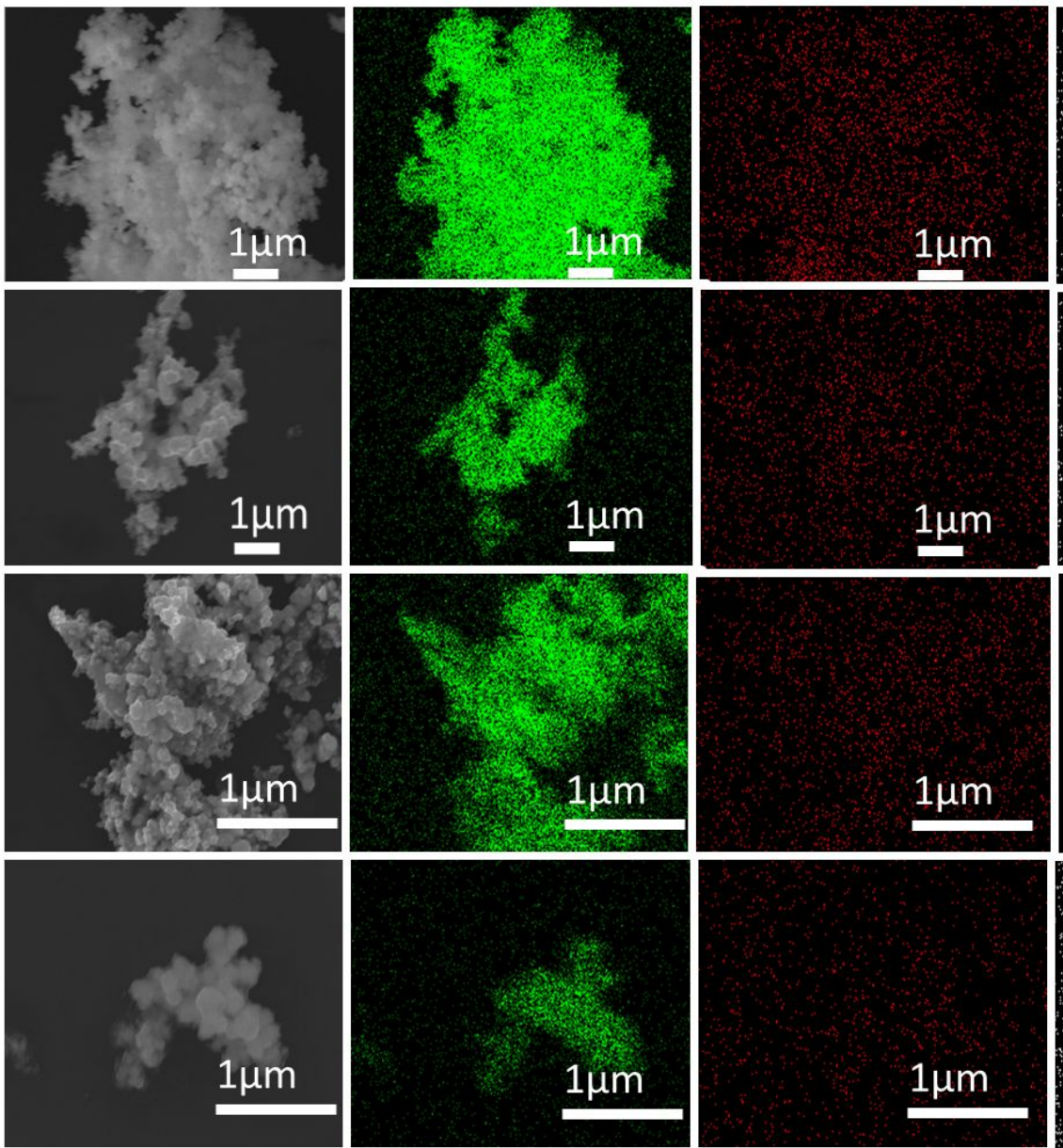

(c)

(d)

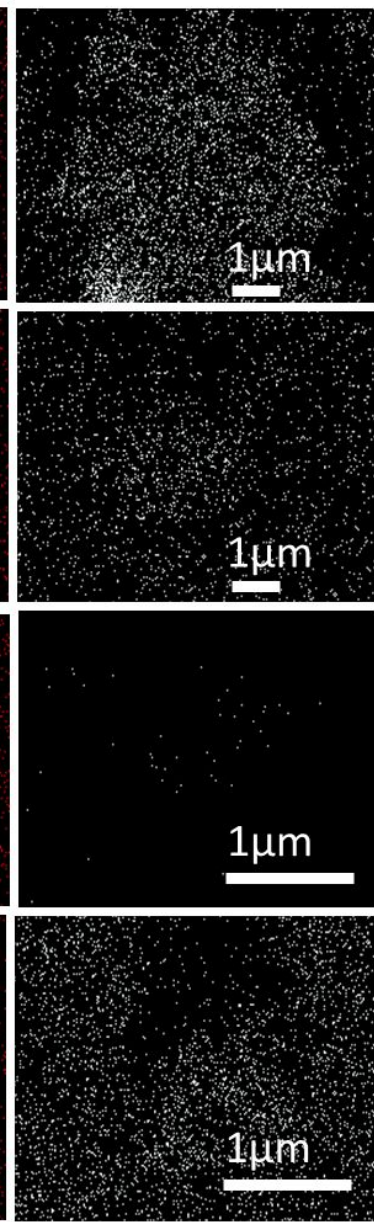

Figure S4. SEM images - Elemental mapping of EuPd-B (1a), EuPd-A (2a), YbPd-B (3a) and YbPd-A (4a). Green colour corresponds to Pd, Red corresponds to RE (1c, 2c = Eu \& $3 \mathrm{c}, 4 \mathrm{c}=\mathrm{Yb})$ and white corresponds to oxygen. 
(a)

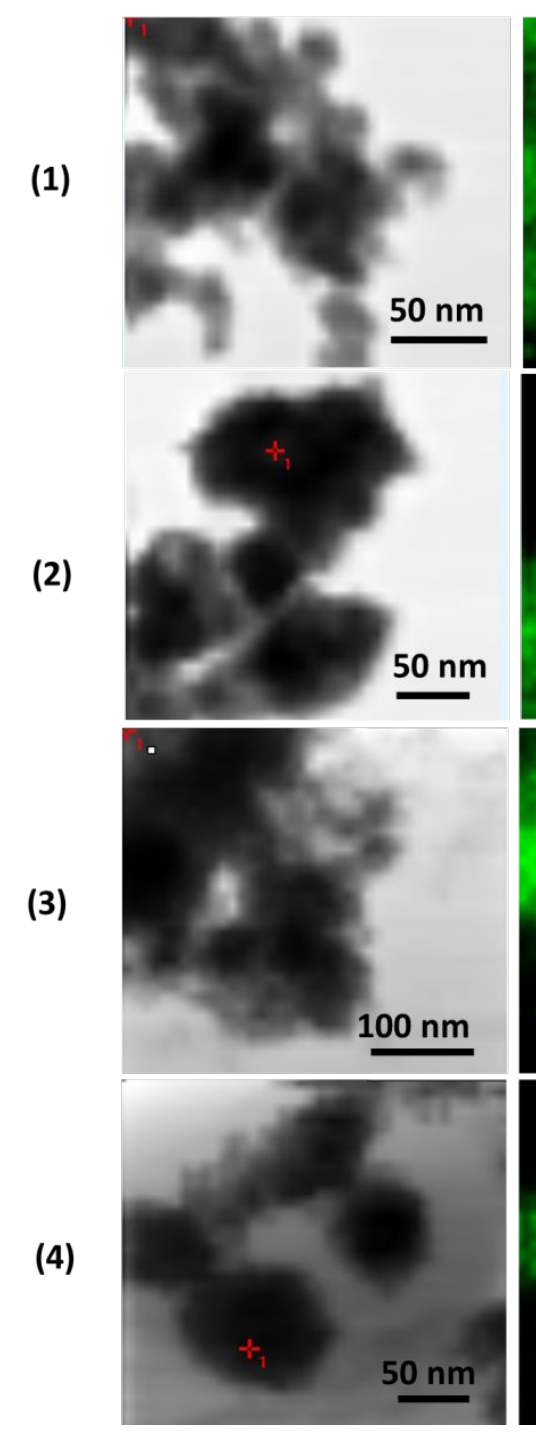

(b)

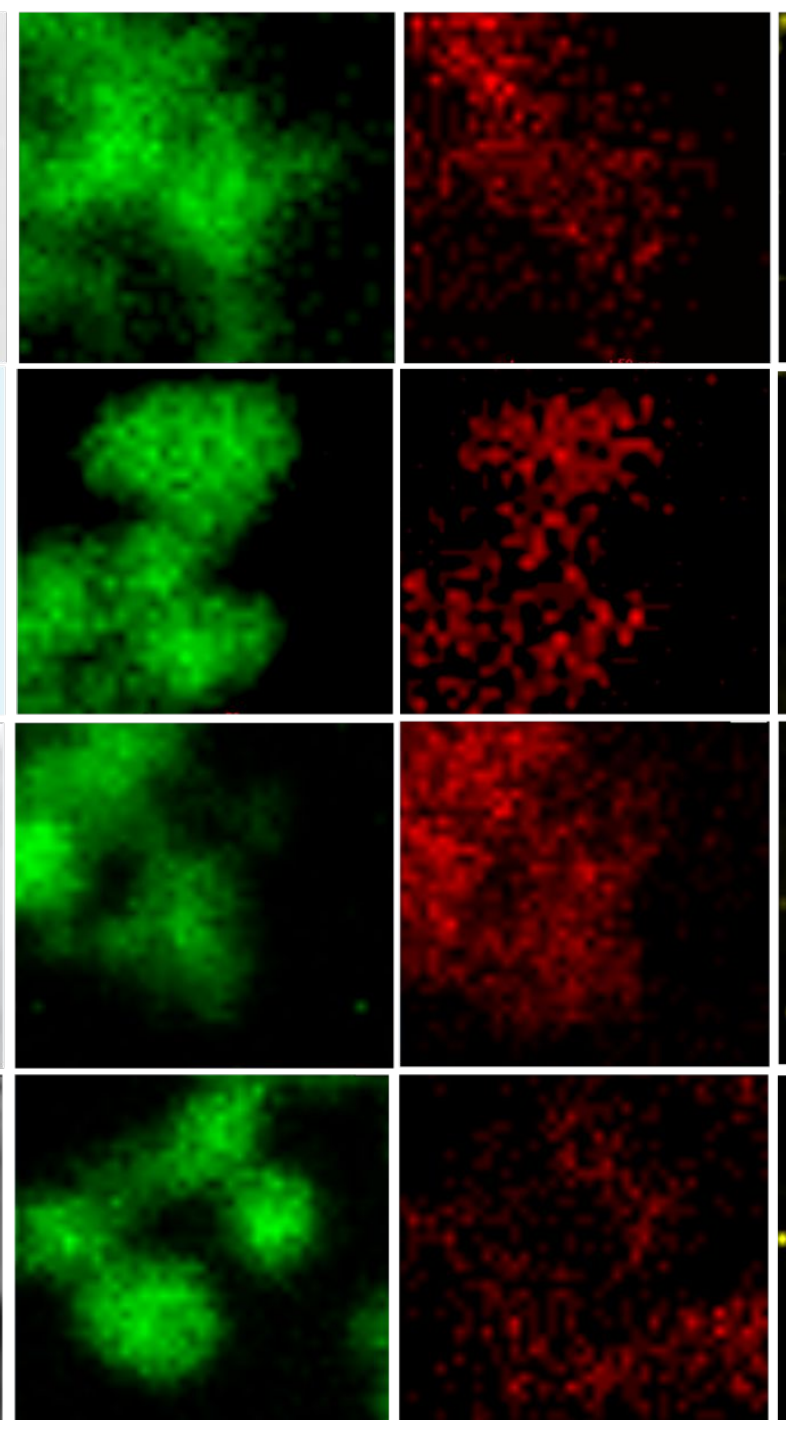

(d)

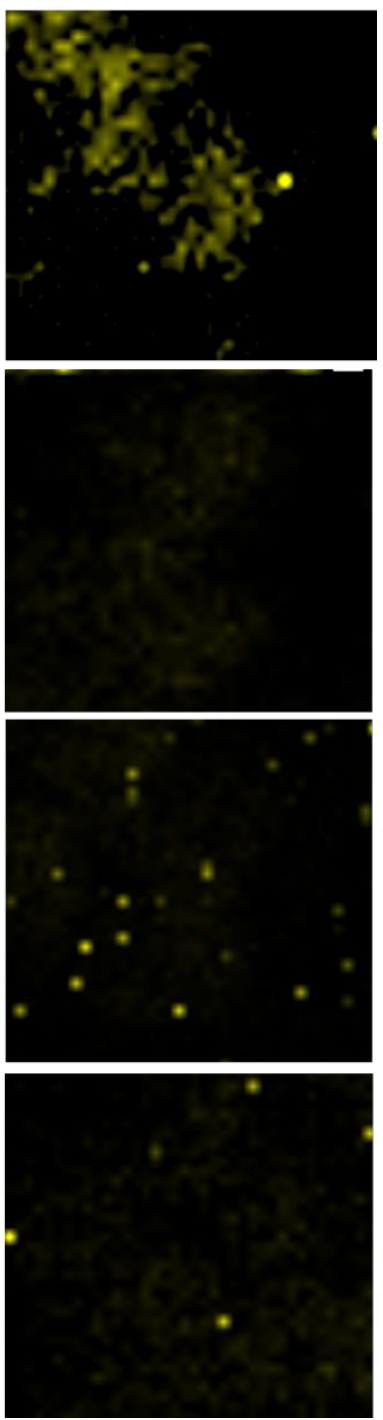

Figure S5. TEM images - Elemental mapping of YbPd-B (1a), EuPd-B (2a), YbPd-A (3a) and EuPd-A (4a). Green colour corresponds to Pd, Red corresponds to RE (1c, 3c = Yb\& 2c, $4 \mathrm{c}=\mathrm{Eu})$ and yellow corresponds to oxygen. 


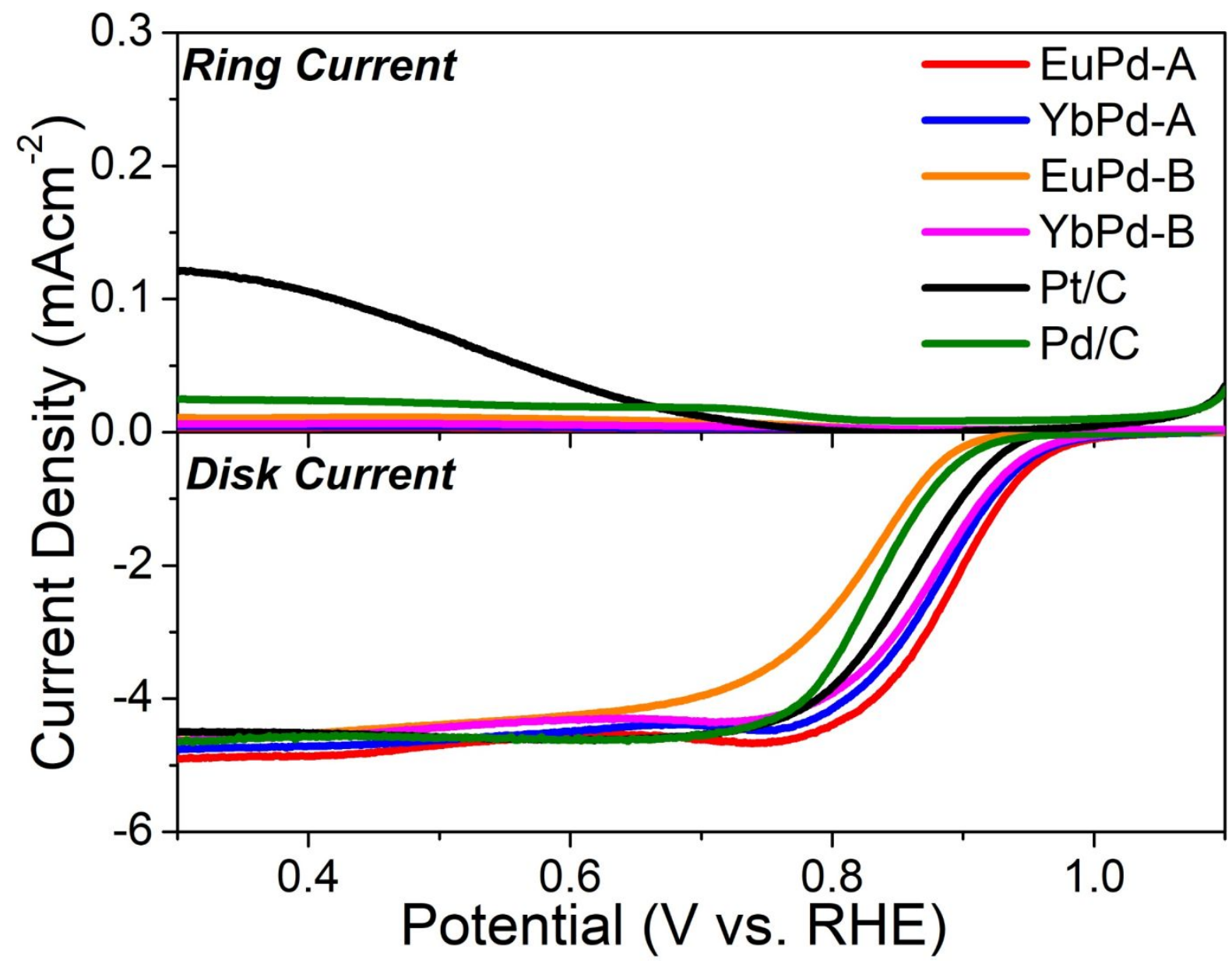

Figure S6. Comparison of RRDE polarization curve of $\mathrm{Pd} / \mathrm{C}(20 \mathrm{wt} \%), \mathrm{Pt} / \mathrm{C}(20 \mathrm{wt} \%)$ and $R E P d$ catalysts recorded at $1600 \mathrm{rpm}$ in $\mathrm{O}_{2}$-saturated $0.1 \mathrm{M} \mathrm{KOH}$. Disk current $\left(\mathrm{I}_{\mathrm{D}}\right)$ represents current due to reduction of $\mathrm{O}_{2}$ to $\mathrm{H}_{2} \mathrm{O}$ and $\mathrm{H}_{2} \mathrm{O}_{2}$ and ring current $\left(\mathrm{I}_{\mathrm{R}}\right)$ signifies current due to oxidation of $\mathrm{H}_{2} \mathrm{O}_{2}$. 


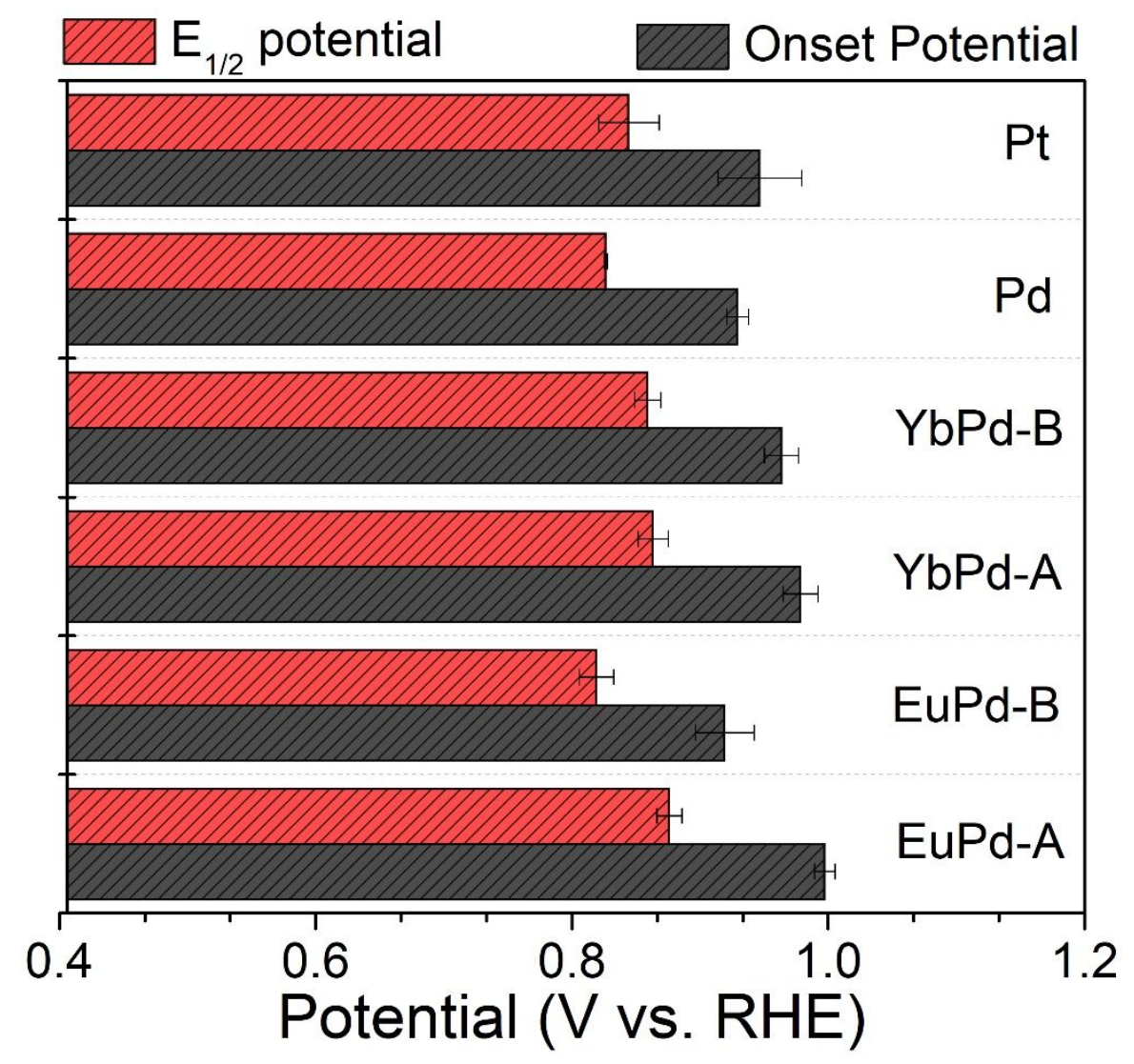

Figure S7. Trend of onset potential and half-wave potentials extracted from the ORR polarization curves along with the standard deviations.
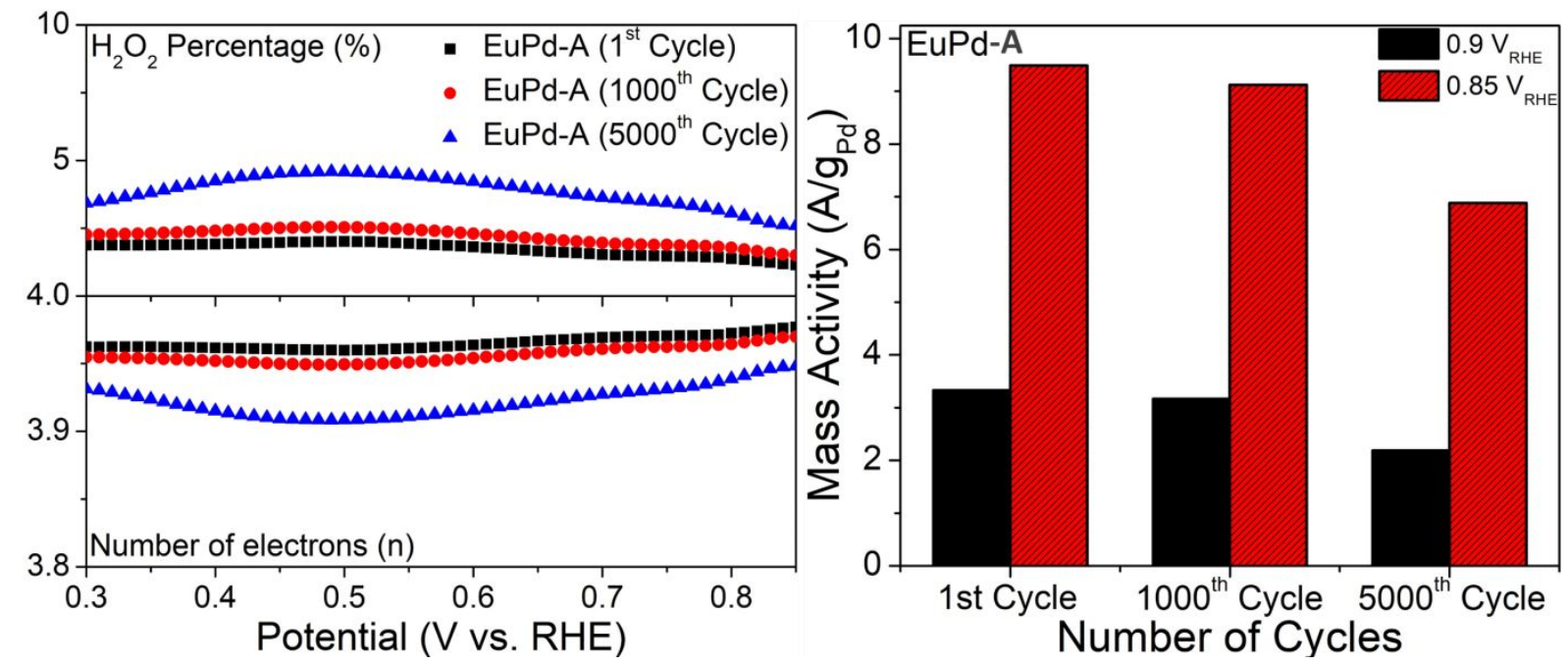

Figure S8. (Left) $\mathrm{H}_{2} \mathrm{O}_{2}$ percentage and number of electrons and (right) mass activity of EuPd-A before and after stability test of 5000 cycles and at a potential of $0.85 \mathrm{~V}$ and $0.90 \mathrm{~V}$ vs. RHE. 


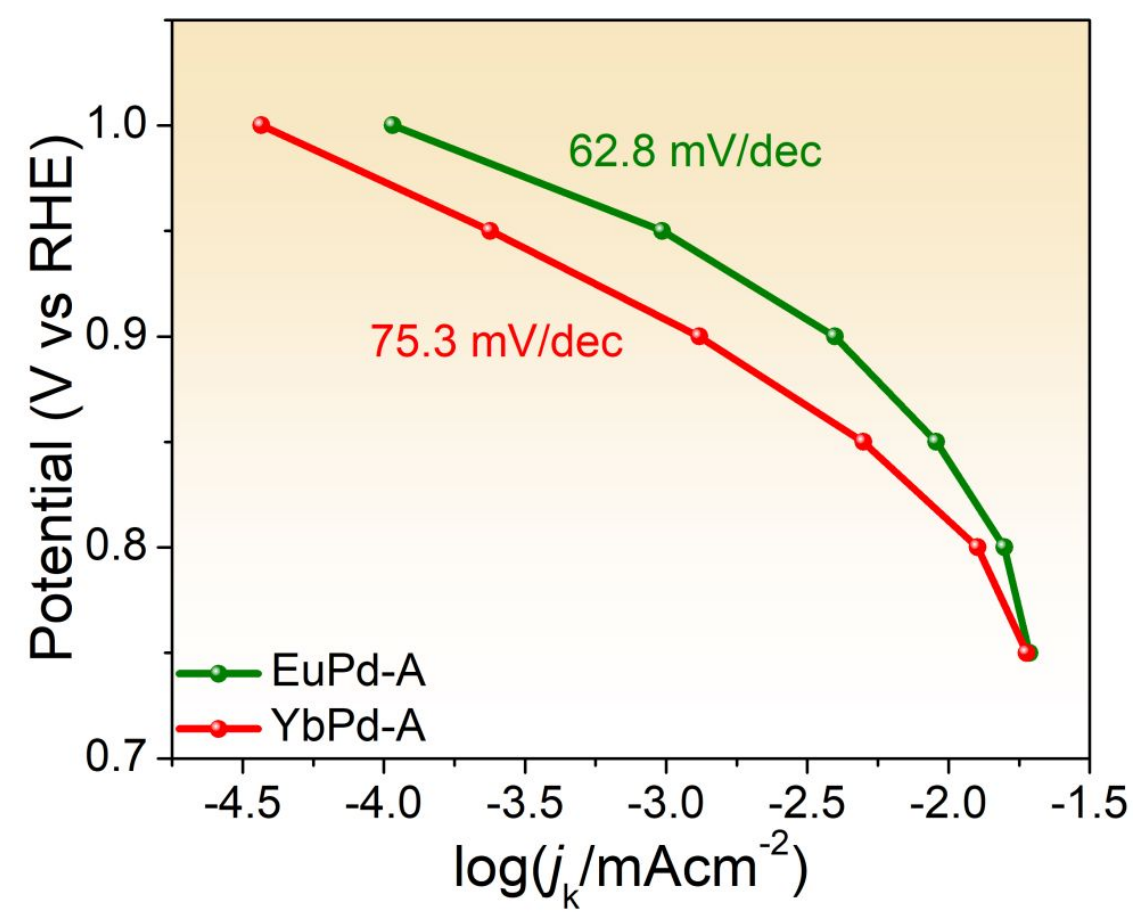

Figure S9. Comparison of Tafel slope of EuPd-A and YbPd-A.

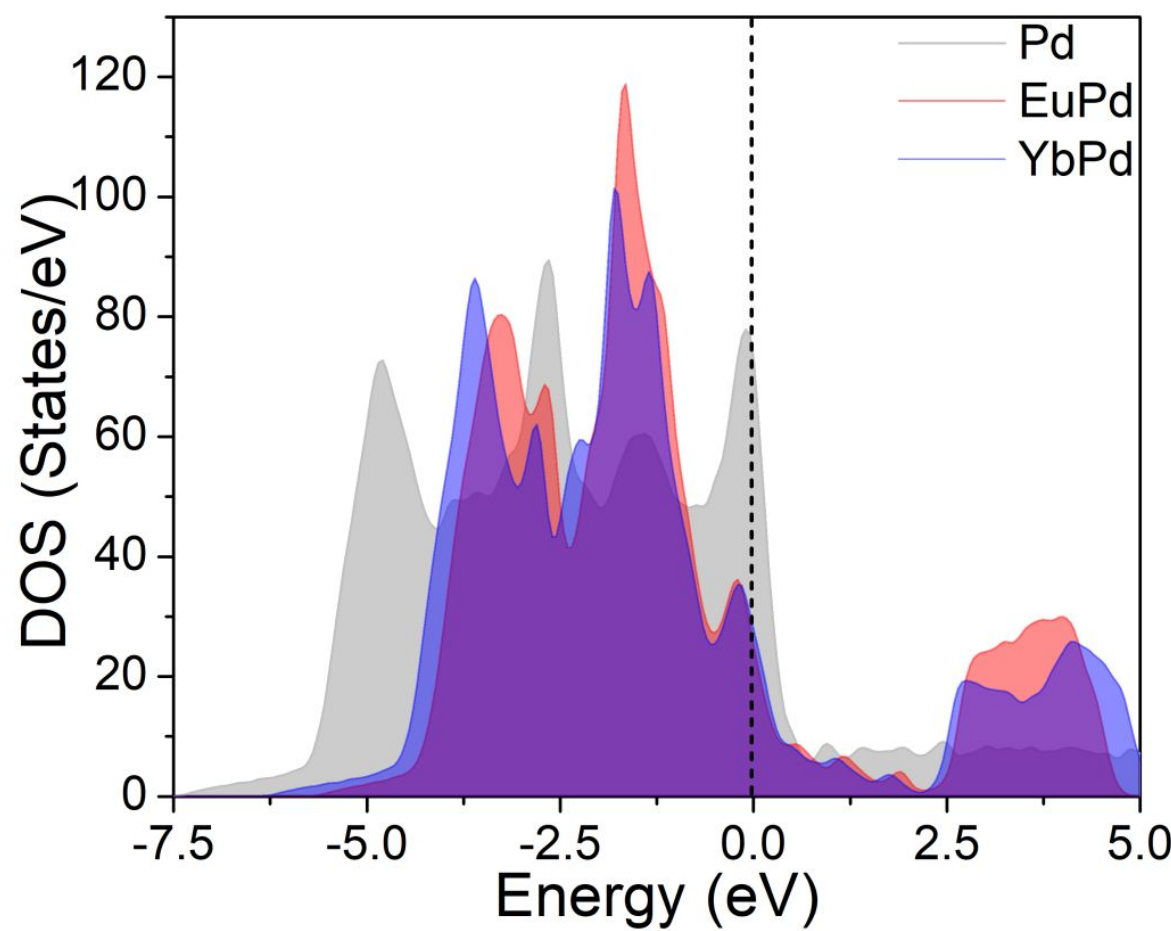

Figure S10. Density of states plot for all the catalyst (Pd, YbPd and EuPd) showing significant amount of DOS at the fermi-level which signifies the metallic behavior of all the catalysts. 

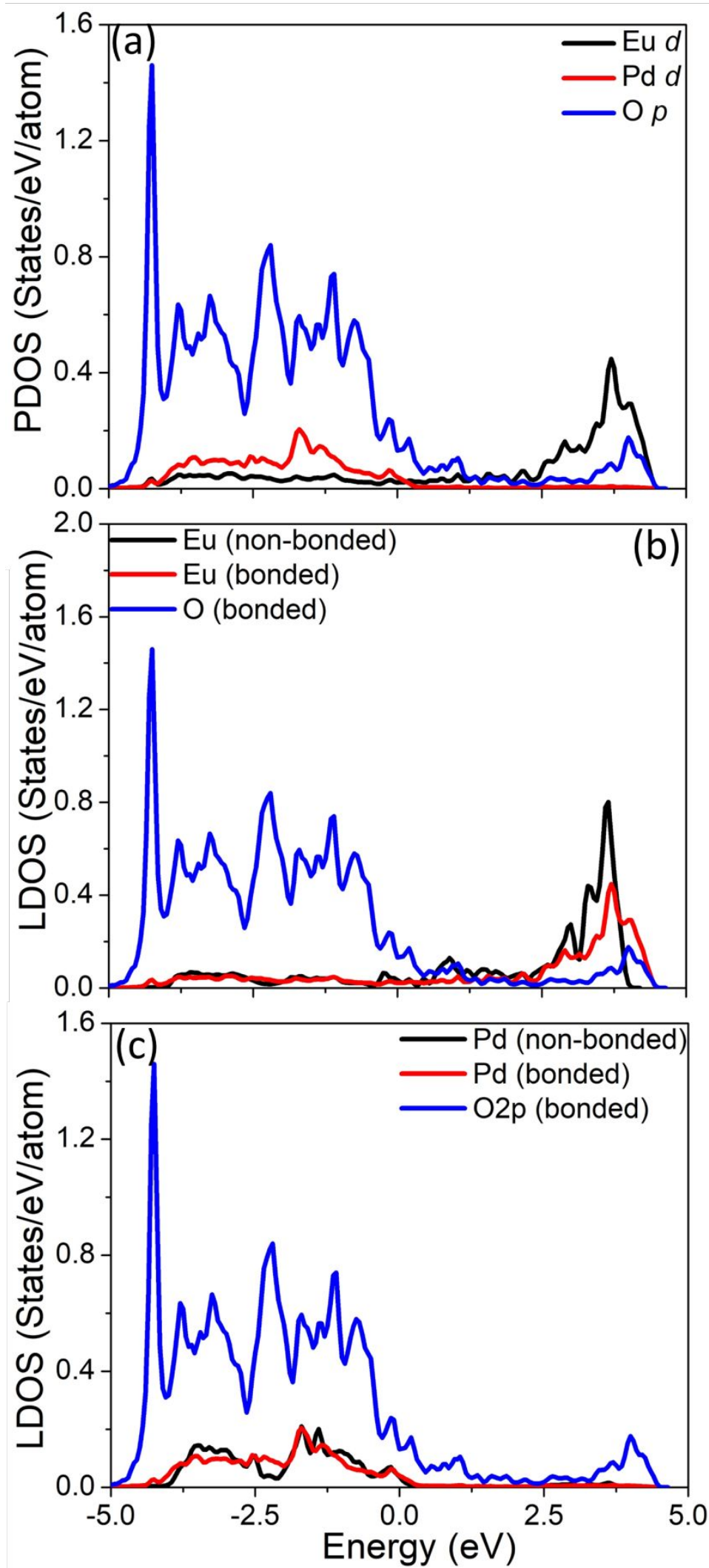

Figure S11. (a) PDOS of Eu- $d$, Pd- $d$ and O-p orbitals showing major contribution of Pd at the valance band and Eu at conduction band and change in LDOS of (b) Eu- $d$ and (c) Pd- $d$ before and after $\mathrm{O}_{2}$ adsorption. 


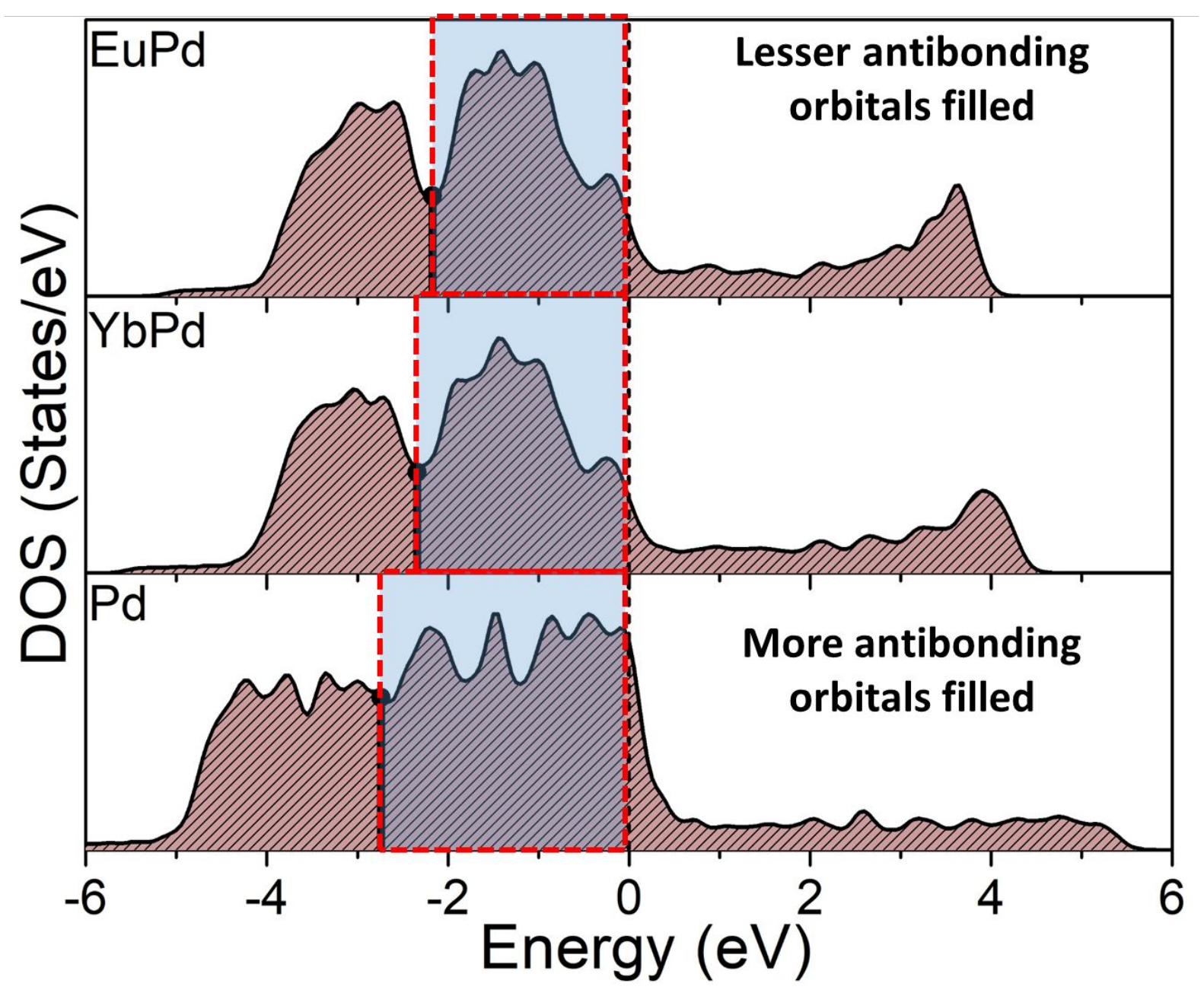

Figure S12. Calculated $d$-band center of YbPd, EuPd and Pd showing the shift in the $d$-band center towards the fermi-level after $\mathrm{Yb}$ and Eu substitution. 

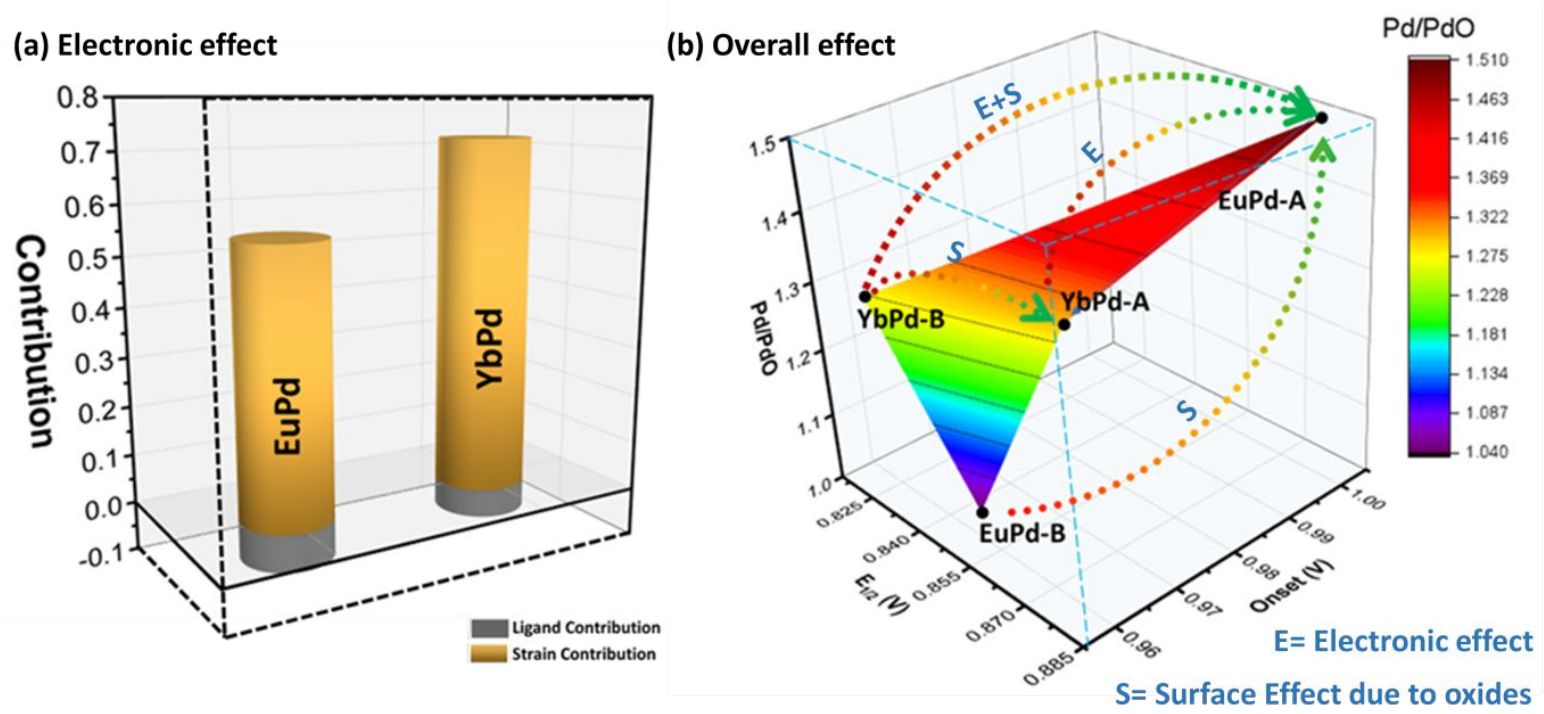

Figure S13. (a) Deconvoluted strain and ligand effect due to $R E$ substitution on Pd lattice showing the dominant effect of strain for both YbPd and EuPd and (b) overall effect of electronic contribution and detrimental effect of surface $\mathrm{PdO}$ on $\mathrm{E}_{\text {onset }}$ and $\mathrm{E}_{1 / 2}$ potentials. EuPd-A, due to lower surface PdO and high lying $d$-orbital, has the highest $\mathrm{E}_{\text {onset }}$ and $\mathrm{E}_{1 / 2}$.
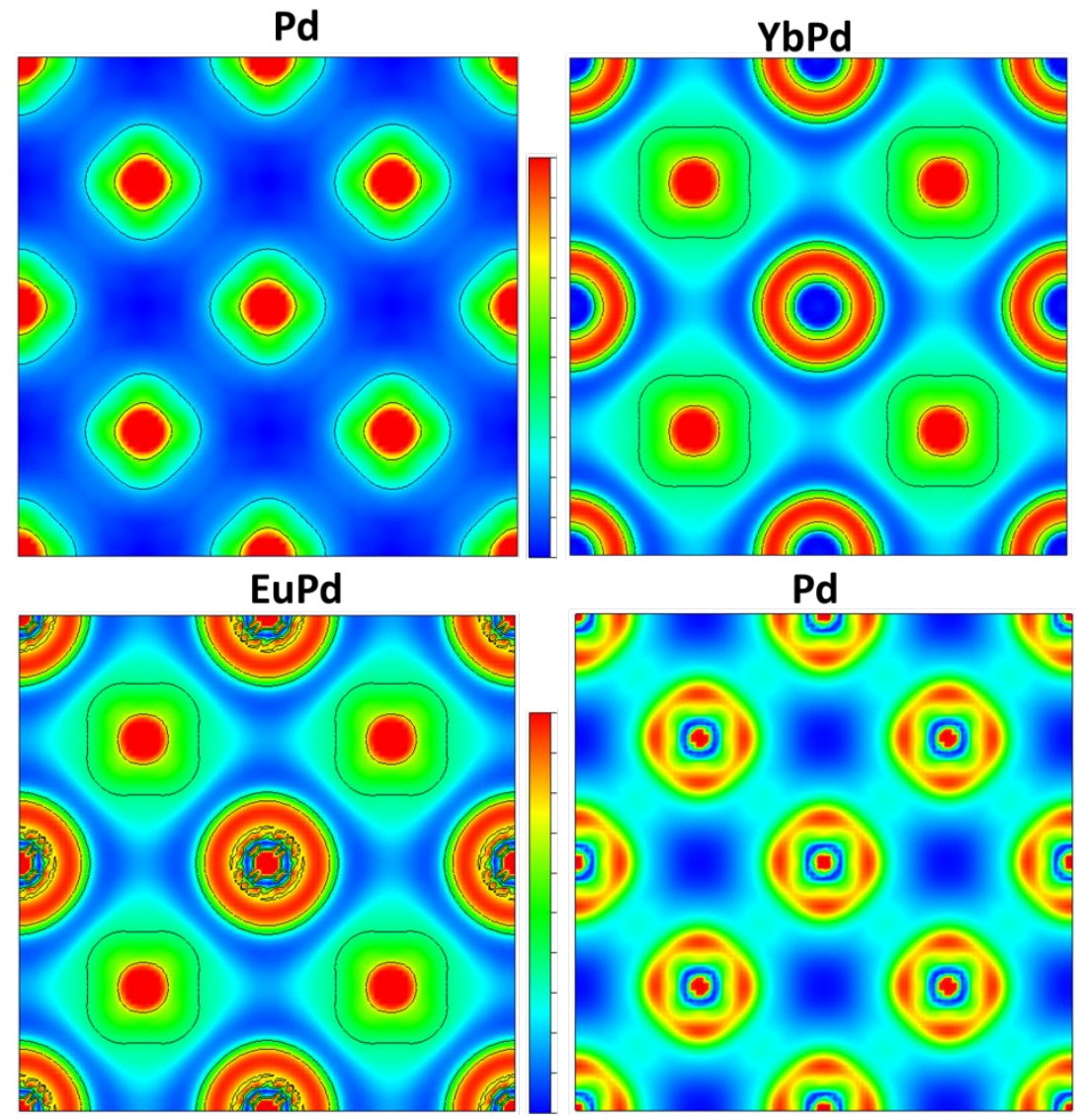

Figure S14. Bonding information in the crystal. 2D-electron localization function mapping of the (100) surface of Pt, YbPd, EuPd and Pd with an isosurface value of 0.8. 


\section{References.}

1. Sarma, S. C.; Subbarao, U.; Khulbe, Y.; Jana, R.; Peter, S. C. Are we underrating rare earths as an electrocatalyst? The effect of their substitution in palladium nanoparticles enhances the activity towards ethanol oxidation reaction. J. Mater. Chem. A 2017, 5 (44), 23369-23381.

2. R. Jiang, D. T. Tran, J. P. McClure and D. Chu, ACS Catalysis, 2014, 4, 25772586. 